\title{
A novel method for evaluating the release of fermentable sugars from cellulosic biomass
}

\author{
Rafael Jimenez-Flores ${ }^{\mathrm{a}}$, Gina Fake ${ }^{\mathrm{b}}$, Jennifer Carroll ${ }^{c}$, Elizabeth Hood ${ }^{\mathrm{d}}$, John Howard ${ }^{\mathrm{b}, *}$ \\ a Dairy Science Department, Dairy Products Technology Center, California Polytechnic State University, San Luis Obispo, CA 93407, United States \\ ${ }^{\mathrm{b}}$ Applied Biotechnology Institute, San Luis Obispo, CA 93430, United States \\ c Department of Chemistry and Biochemistry, California Polytechnic State University, San Luis Obispo, CA 93407, United States \\ d Arkansas State University, State University, AR 72467, United States
}

\begin{abstract}
A B S T R A C T
Efficient hydrolysis of cellulosic biomass into free sugars for the production of bioethanol would allow utilization of an abundant and renewable natural resource. However, complex microcrystalline cellulose in biomass resists digestion. A preferred method to deconstruct cellulose is using enzyme cocktails, but the presence of multiple enzymes with synergistic outcomes for this process complicates direct biochemical analysis as a reliable indicator of deconstruction. Methods to detect release of free sugars rather than direct measurement of enzymatic activity are available but are time-consuming, and do not reflect the production of metabolic inhibitors of microbial growth which may interfere with the subsequent fermentation of sugars to ethanol. The work presented here describes an automated method for detecting the release of free sugars from enzymatic digestion of cellulosic biomass that allows for realtime measurements during the enzyme treatments and provides an indication of metabolic inhibitors that can interfere with subsequent growth of microbes during fermentation.
\end{abstract}

\section{Introduction}

Plant biomass is a complex matrix of polymers comprising the polysaccharides cellulose, hemicellulose, and lignin as the major structural components. Cellulose, the most abundant biopolymer on earth, is a simple, linear polymer of glucose. However, its semi-crystalline structure is notoriously resistant to hydrolysis by both enzymatic and chemical means. Any strategy designed to use cellulosic material for feed or fuel must incorporate the ability to efficiently convert the polysaccharide components of plant cell walls to simple sugars and then to ethanol by fermentation.

A preferred method for the digestion of cellulose is accomplished by heat and chemical pretreatment followed by the use of enzymes [1]. There are a number of enzymes required to break down cellulose that work in synergy including endo-cellulase, exocellulase and glucosidase [2,3]. Other proteins, such as expansins, may be helpful in facilitating the breakdown process [4]. Cellulases are a subset of the glycosyl hydrolase superfamily of enzymes that have been grouped into more than 100 families based on amino acid sequence similarity, enzyme reaction mechanism, and protein fold motif [5]. Endo- and exo-cellulases are two classes of cellulases that have been shown to be effective in the hydrolysis of cellulose from biomass [6].

The current impetus to increase the use of biofuels to offset the dependency on foreign oil has catalyzed efforts to improve feedstock, isolate or engineer new enzymes, co-cultures and microorganisms that will be more efficient in breaking down cellulose. To expedite these discoveries and improvements, it would be valuable to have an assay that could be automated, was flexible and reliable and easy to use. The complex nature of microcrystalline cellulose, and the fact that different proteins can work synergistically but may vary in activity on the particular type of cellulose substrate $[7,8]$, make this a challenge. Furthermore, different enzymes may function at different stages of degradation. Real-time analysis would be required to optimize degradation due to these effects.

Several different assay methods have been previously reviewed [9-11]. Early methods used filter paper as the source of cellulose and changes in viscosity were measured to infer release of free sugars. Other assay methods have been developed, including microplate filter paper assays [12], monitoring the release of dyes incorporated into a cellulosic substrate [13,14], or using chemical analysis to detect the release of free sugars from cellulose [15]. While free sugar detection by chemical analysis is arguably one of the most reliable methods, it is cumbersome and time-consuming, 
thereby limiting the number of samples that can be easily tested with different combinations of enzymes, microorganisms and substrates.

The difficulty in using these analytical methods for large-scale studies has led to investigations of alternative processes with the potential for high throughput such as enzyme assays [16-18] and immunochemical assays [19]. Gel electrophoresis has also been used to identify the enzymes involved [20] but this method is cumbersome for high-throughput screening. While having utility for determining the concentrations of specific enzymes, these methods cannot by themselves be used to evaluate the efficiency of the release of free sugars. Since multiple enzymes are required, each with different catalytic activities, these preceding assays do not measure synergistic outcomes. In addition, assays of the degradation of simple biochemical substrates do not necessarily indicate the effectiveness of cellulases on the microcrystalline cellulose found in biomass [21].

Microbial colonies producing cellulose-degrading enzymes have been identified by growth on solid media containing cellulose as the only carbon source [22]. In this work, we report the use of the microbial approach taken one step further by developing a method that measures growth of microbes on cellulosic substrates as a measure of the release of free sugars. By coupling a microbial growth assay directly to the enzymatic digestion of cellulose we can easily monitor digestion of cellulose in real time and without additional effort. This assay also indicates whether metabolites are generated that can affect downstream utilization of those sugars by microorganisms. Using a commercially available microbial growth detection system (e.g. BacT/Alert 3D, bioMérieux, France), changes in microbial growth can be correlated with free sugar release. The system we used measures optical density based on the production of $\mathrm{CO}_{2}$ and $\mathrm{pH}$. In this case turbid samples do not confuse results, which is important for biomass testing, as cellulose is insoluble. The equipment is automated, applicable to a large range of microorganisms, allows the course of the reaction to be followed in real time, and permits intervention at any point. Significantly, this system verifies that reaction products are compatible with microbial growth, thus providing an indicator that not only are sufficient free sugars being made, but that metabolites being produced do not interfere with subsequent microbial growth, such as is required for fermentation. The results presented below illustrate the adaptability of these growth assays to a completely new field for assessing biomass degradation using different enzyme combinations, substrates and organisms. Rather than sampling and testing degradation products by recovery and assay at discrete times, this system provides a functional assessment of the ability of the degradation products of cellulose to support growth of fermentative yeast. The opportunity to manipulate individual components provides a useful tool for screening novel and combinations of enzymes and organisms for optimizing the conversion of cellulose into biofuels.

\section{Materials and methods}

\subsubsection{Yeast growth assay}

A starter culture of Saccharomyces cerevisiae was allowed to grow in $5 \mathrm{~mL}$ of yeast broth on a rotary shaker at $37^{\circ} \mathrm{C}$ and $225 \mathrm{rpm}$ for $4-6 \mathrm{~h}$ to reach an $\mathrm{OD}_{550}=0.5$ when diluted 60 -fold. $0.1 \mathrm{~mL}$ of the culture was used to inoculate BacT bottles containing $6.25 \mathrm{mg} / \mathrm{mL}$ cellulose suspended in $40 \mathrm{~mL}$ of $140 \mathrm{mM}$ citrate $/ 90 \mathrm{mM}$ bicarbonate buffer, pH 5 . All reagents were autoclaved or filter-sterilized and the assay was carried out under sterile conditions. Cellulase was added last to the BacT bottle to initiate the reaction and a rubber stopper was inserted. $180 \mathrm{~mL}$ of atmosphere in the bottle was aseptically removed with a syringe, rendering the growth assay anaerobic. Samples were maintained at $37.5^{\circ} \mathrm{C}$ and rocked at 70 times per minute. OD values based on $\mathrm{CO}_{2}$ and $\mathrm{pH}$ changes were automatically recorded every $10 \mathrm{~min}$ on the BacT/Alert machine.

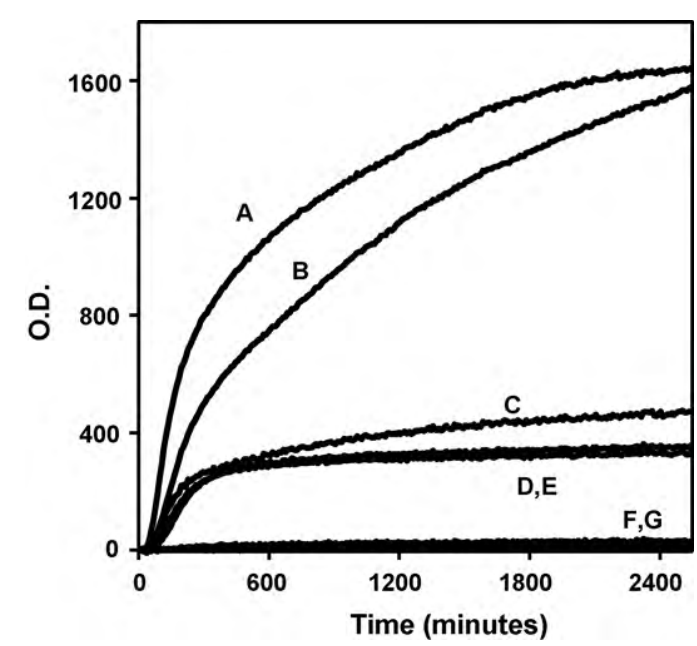

Fig. 1. Comparison of yeast growth on glucose or products of enzymatic cellulose degradation. Growth of yeast on medium supplemented with glucose was compared to growth on products of cellulase-mediated digestion of cellulose in a final assay volume of $40 \mathrm{~mL}$ as described in Section 2. The treatments were: A: yeast + cellulose $+2 \%$ glucose; $B$ : yeast + cellulose + cellulase; $C$ : yeast + cellulase; $D$ : yeast + cellulose; E: yeast only; F: cellulose + cellulase; G: cellulase only. Growth of yeast is reported as OD based on readings from the BacT/Alert system over a total of $2400 \mathrm{~min}(40 \mathrm{~h})$. Note that growth in treatment B (yeast + cellulose + enzyme) is similar to growth in treatment $\mathrm{A}$ (yeast + cellulose $+2 \%$ glucose).

\subsubsection{Rumen fluid}

To obtain rumen microorganisms, $3 \mathrm{~L}$ of rumen fluid were collected from a freshly culled dairy cow by abattoir staff (Central Valley Meat Company, Inc., Hanford, CA). The rumen fluid was immediately placed on dry ice and later frozen at $-50^{\circ} \mathrm{C}$. It was thawed on ice, divided into $1 \mathrm{~mL}$ aliquots, and returned to $-50{ }^{\circ} \mathrm{C}$ for later use.

\subsubsection{Cellulose substrates}

The sources of cellulose used were Sigmacell (Sigma Chemical Co., St. Louis, MO), $1 \mathrm{~mm} \times 1 \mathrm{~mm}$ pieces of Whatman paper \#1, pretreated rice hulls (FutureFuel Chemical Company (FFCC), Batesville, AR), and pretreated hardwood (FFCC). For each experiment a $40 \mathrm{~mL}$ final assay volume with a concentration of $6.25 \mathrm{mg} / \mathrm{mL}$ cellulose substrate in $140 \mathrm{mM}$ citrate/90 mM bicarbonate buffer, $\mathrm{pH} \mathrm{5}$, was used.

\subsubsection{Cellulase}

Cellulase (Spezyme CP, Genencor) was used for all cellulose digestion assays with the exception of those using Trichoderma reesei (Sigma \# C8546) that was used for a comparison study. The Spezyme CP mixture is provided as a liquid and it was not possible for us to determine the exact amount of each of the various enzymes in the cocktail, therefore we used volume in $\mu \mathrm{L}$ of cellulase as it relates directly to the product. In the case of $T$. reesei, $\mathrm{mg}$ of cellulase are given corresponding to the dry weight of the product. Cellulase enzymatic assays were performed as described earlier [23] at $50^{\circ} \mathrm{C}$ using T. reesei (Sigma \# C8546) as the standard to compare enzyme activity.

\subsection{Free sugar analysis}

Free sugar concentrations were measured using protocols established by the National Renewable Energy Laboratory Technical Report, NREL/TP-510-42623 (http://www.nrel.gov/biomass/pdfs/42623.pdf). Chemical analysis for sugars was performed on a Shimadzu Prominence Series HPLC with a Bio-Rad Aminex (HPX87P) column, a Bio-Rad de-ashing pre-column and an Agilent 1200 Series Refractive Index Detector. Results shown are the median of three replicate samples.

\section{Results}

To establish a baseline for yeast growth using the BacT/Alert system, samples were prepared as described in Section 2 and supplemented with $2 \%$ glucose to obtain optimal growth. Fig. 1 (line A) shows a typical growth response over time with OD reaching maximum values by $2400 \mathrm{~min}$ ( $40 \mathrm{~h}$ ). Controls using cellulose as carbon source supplemented with $10 \mu \mathrm{L}$ cellulase resulted in growth den- 


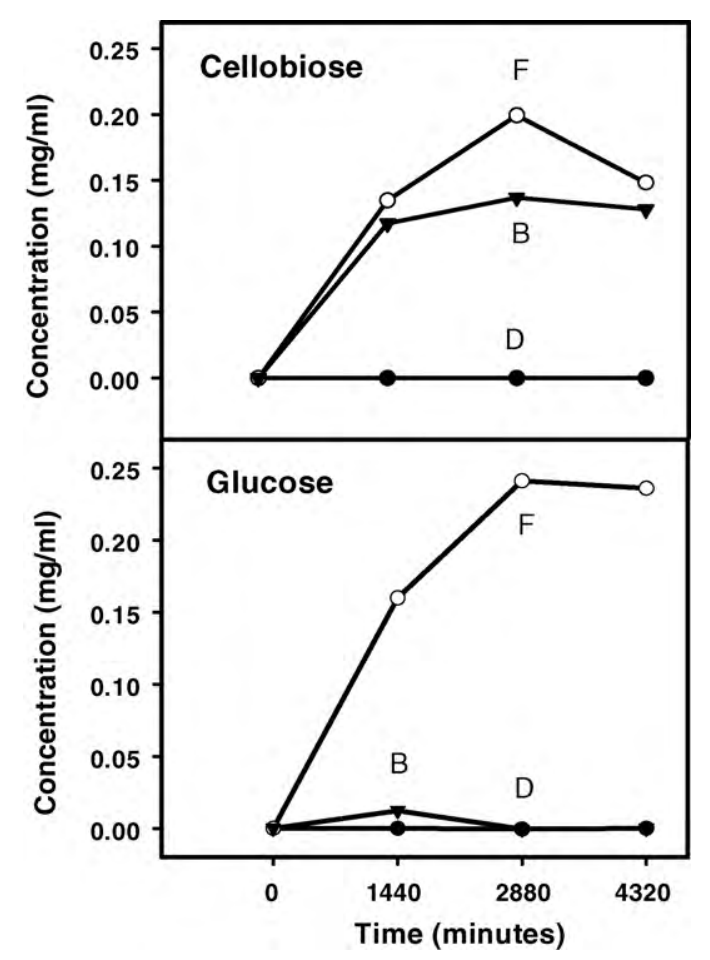

Fig. 2. HPLC analysis of free sugars released by enzymatic degradation of cellulose Samples of assay medium from various treatments taken at 24-h time points ( 1440 , 2880 and $4320 \mathrm{~min}$ ) were analyzed by HPLC for the presence of free sugars. Only cellobiose and glucose were reproducibly detected above $0.01 \mathrm{mg} / \mathrm{mL}$. The treatments were: closed circles D: yeast + cellulose; open circles F: cellulose + cellulase; triangles B: yeast + cellulose + cellulase. Note that yeast consumes glucose, but not cellobiose (triangles).

sities approaching that of the yeast grown on glucose (Fig. 1, line B). When the medium contained cellulase but no cellulose (Fig. 1C); cellulose but no cellulase (Fig. 1, line D) or neither cellulose nor cellulase (Fig. 1, line E); only a small amount of growth is seen in samples C, D and E in the first $300 \mathrm{~min}(5 \mathrm{~h})$. This is most likely due to residual sugar from the yeast starter culture used as inoculum. Treatments that do not have yeast but which contain either cellulose plus cellulase (Fig. 1, line F) or cellulase alone (Fig. 1, line G) show the predicted baseline values with no growth. Therefore the use of yeast growth as an indicator appears to provide an effective and qualitative assay for the release of free sugars.

Independent validation of sugar release using chemical analysis was performed on the growth treatments above. Aliquots from treatments B (yeast + cellulose + cellulase), C (yeast + cellulase, no cellulose) and $\mathrm{F}$ (cellulase + cellulose, no yeast), were taken at various times over a 72-h period and analyzed for free sugars by HPLC. The results, in Fig. 2, show that cellulose digestion in the absence of yeast (treatment F) releases cellobiose and glucose, as expected (Fig. 2, open circles). In treatment $\mathrm{D}$ containing yeast + cellulose but no cellulase, no sugars were detected (Fig. 2, closed circles). Treatment $B$ containing yeast + cellulose + cellulase showed the presence of cellobiose but almost no glucose (Fig. 2, triangles), indicating that the yeast was utilizing glucose as fast as it was being released, but not cellobiose. No other sugars were reproducibly detected above a concentration of $0.01 \mathrm{mg} / \mathrm{mL}$. These results validate the BacT/Alert system as a predictor of sugar release and the ability of yeast to grow on the reaction products providing a qualitative measure of cellulose breakdown.

The BacT/Alert assay could potentially also be used to quantify the effectiveness of different cellulase mixtures; a single enzyme's effectiveness in a mixture of cellulases; or to examine synergistic effects of different proteins with cellulase. To measure quantitative
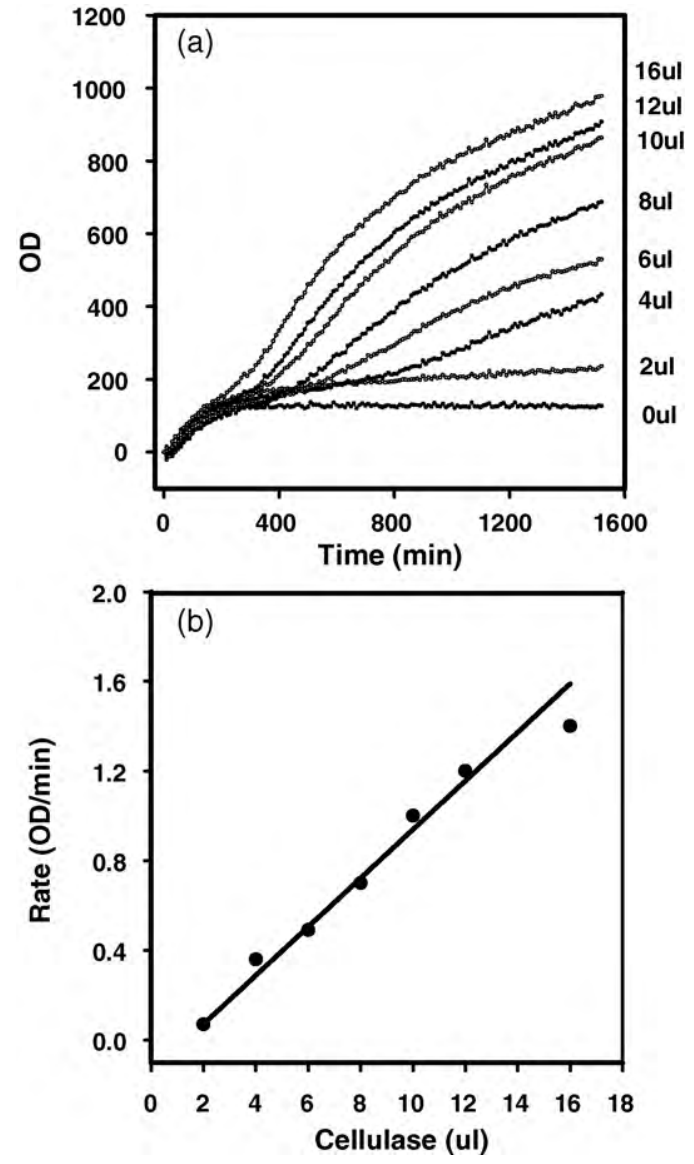

Fig. 3. (a) Dependence of yeast growth on cellulase concentration. Different concentrations of cellulase were added to the assay medium, and yeast growth monitored by periodic OD measurements. The treatments were $16 \mu \mathrm{L} ; 12 \mu \mathrm{L} ; 10 \mu \mathrm{L} ; 8 \mu \mathrm{L} ; 6 \mu \mathrm{L}$; $4 \mu \mathrm{L} ; 2 \mu \mathrm{L}$; and $0 \mu \mathrm{L}$ with sampling over $1440 \mathrm{~min}(24 \mathrm{~h})$. It can be seen that growth is directly proportional to cellulase concentration. (b) Rate of yeast growth at $12 \mathrm{~h}$ as a function of enzyme concentration. The rate of yeast growth was calculated by taking the slope of the line (OD/min) over the first $720 \mathrm{~min}(12 \mathrm{~h})$ for the various concentrations of cellulase in (a). This rate was then plotted against enzyme concentration which provided the linear relationship shown here, indicating that cellulose degradation is directly proportional to enzyme concentration.

outcomes, we tested cellulase mixtures at various concentrations $(1-16 \mu \mathrm{L})$ with yeast growth as an indicator of enzyme activity (Fig. 3). The results show that yeast growth is dependent on enzyme concentration over $20 \mathrm{~h}$ (Fig. 3a) and these data can also be used to establish a quantitative relationship by examining the final OD at $40 \mathrm{~h}$ (data not shown). The concentration of enzyme vs. rate of yeast growth in the first $12 \mathrm{~h}$ shows a linear relationship (Fig. $3 \mathrm{~b}$ ) and provides a quick and useful indicator of sugar release.

In order to confirm that the relationship between enzyme concentration and yeast growth was not unique to one specific cellulase mixture, we repeated the above treatments using a different source of cellulase (T. reesei cellulase, Sigma). The result in Fig. 4 demonstrates that a direct relationship between enzyme concentration and growth is maintained with a different enzyme mixture.

We next evaluated the potential of this system to monitor degradation of different sources of cellulose. In addition to our standard assay substrate, Sigmacell, Whatman paper \#1, and the abundant cellulosic ethanol substrates, pretreated rice hulls and hardwood, were used. Fig. 5 shows the yeast growth-supporting ability of these cellulose sources when treated with cellulase. All of the cellulose sources supported yeast growth directly proportional to the amount of enzyme added. However, different sources of cellulose required different amounts of cellulase to obtain the 


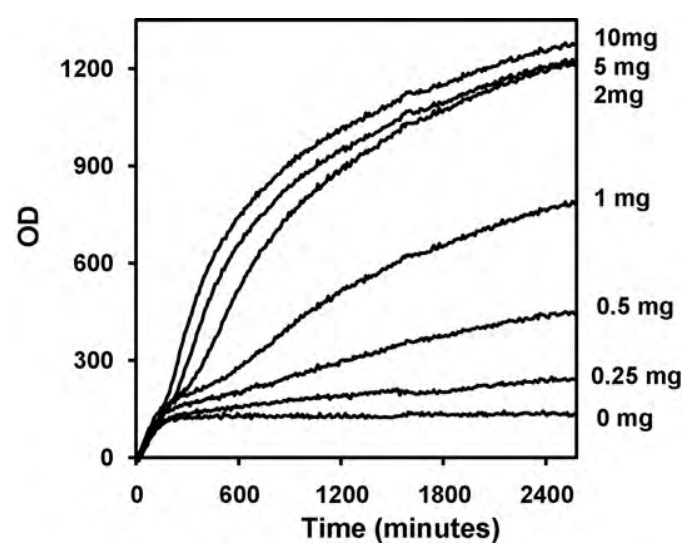

Fig. 4. Growth dependence of yeast on $T$. reesei cellulase concentration. Varying amounts ( $0 \mathrm{mg}, 0.25 \mathrm{mg}, 0.5 \mathrm{mg}, 1 \mathrm{mg}, 2 \mathrm{mg}, 5 \mathrm{mg}$ and $10 \mathrm{mg}$ ) of $T$. reesei cellulase were added to the assay medium. Yeast growth was monitored over $2400 \mathrm{~min}$ $(40 \mathrm{~h})$. The graph shows that growth of yeast is proportional to $T$. reesei cellulase concentration up to around $2 \mathrm{mg}$ cellulase.

same amount of yeast growth, showing different amenability to enzymatic hydrolysis. Using Fig. 5, we estimated the cellulase concentration needed to obtain $50 \%$ digestion $\left(D_{50}\right)$ for each type of substrate to compare relative digestibility of the cellulose source by this enzyme mixture. Sigmacell required the lowest concentration of enzymes to permit yeast growth ( $\mathrm{DC}_{50}=2 \mu \mathrm{L}$; data not shown) followed by Whatman paper and hardwood $\left(\mathrm{DC}_{50}=4 \mu \mathrm{L}\right)$. Rice hulls required the highest concentration of enzymes $\left(D_{50}=40 \mu \mathrm{L}\right)$, probably due to the presence of silica in rice hulls, which interferes with cellulose digestion [24]. This method may aid in evaluating the best enzyme cocktails for specific cellulose substrates to determine the relative digestibility, providing utility not only for comparing digestibility of different plant species, but also modified cellulose products from the same plant species, either by screening germplasm or by engineering enzymes into the plant as has been suggested by others [25].

Growth of $S$. cerevisiae is a dependable and accessible indicator for routine tests but we also wanted to assess if this method was applicable to other microbial cultures grown on cellulose. Many microorganisms are under investigation for their ability to grow on sugars other than glucose. These cultures may be more efficient if they can also use additional sugars [26,27], to increase the overall efficiency of ethanol formation. There is also an interest in performing simultaneous saccharification and fermentation [28,29]. Using microbial growth as an indicator requires not only the release of free sugars, but ensures that reaction products do not interfere with growth.

One of the most complex natural cellulose-digesting ecosystems exists in the rumen fluid of cows [30]. Although rumen microorganisms are capable of digesting cellulose, exogenous cellulase added to animal feed can increase the utilization of cellulosic feedstocks even in ruminant animals [31]. Therefore, while rumen microorganisms are able to convert the cellulosic material into free sugars, they cannot do this completely. This system may be used to study the growth of rumen microorganisms with and without exogenous cellulases. Rumen fluid was therefore used in place of yeast and the growth of microorganisms was measured in the BacT/ALERT in media with cellulose. Fig. 6 illustrates that there was growth of the rumen microorganisms on minimal media with cellulose albeit to much lower ODs than those obtained in media with glucose. However, when exogenous cellulase was added, growth increased dramatically indicating that while the microorganisms can grow on cellulose as a carbon source, growth is limited by the amount of enzyme available to break down cellulose and provide fermentable sugars. There appears to be a multiphase curve, which may result
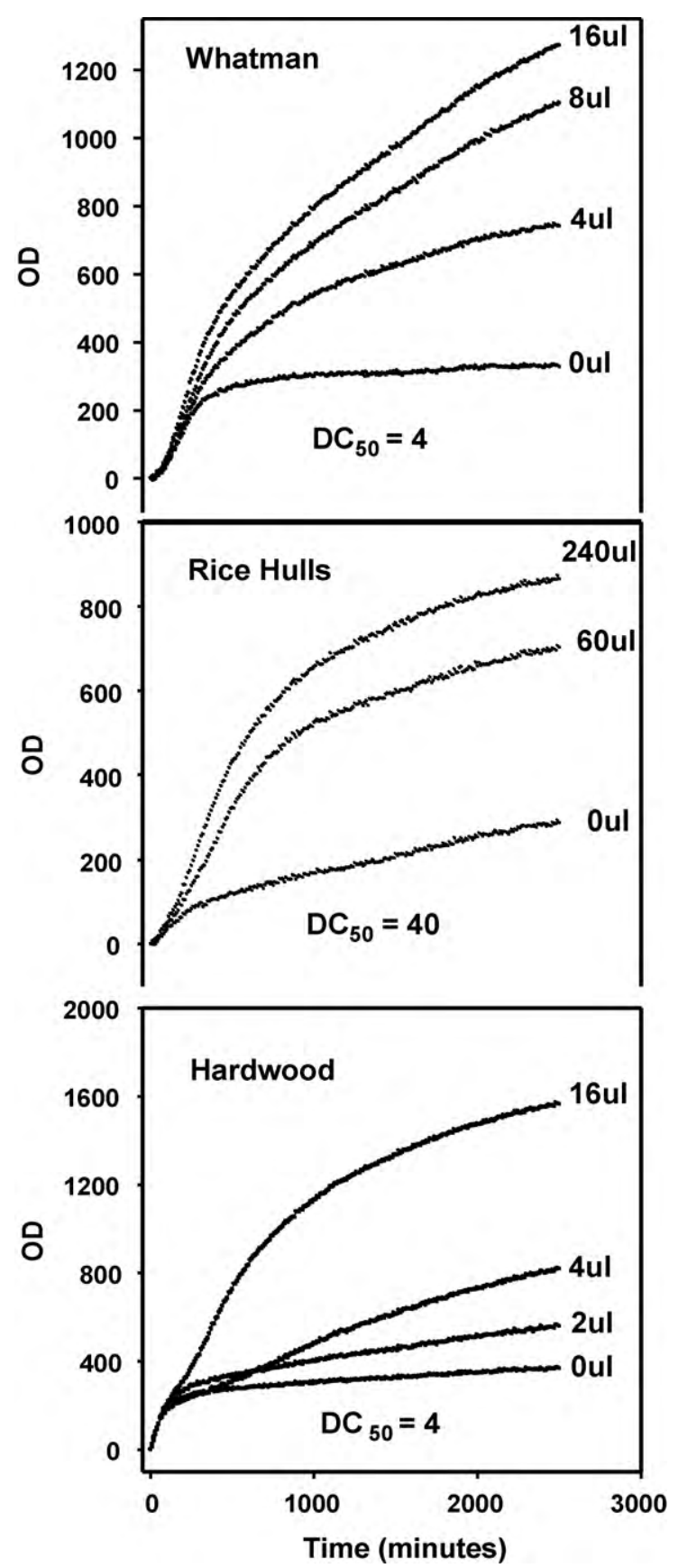

Fig. 5. Growth of yeast on alternative cellulosic substrates. Varying cellulase concentrations as indicated on the graph were tested for yeast growth over a $3000 \mathrm{~min}$ $(50 \mathrm{~h})$ period using different cellulosic substrates: Whatman paper \#1; pretreated rice hulls or pretreated hardwood. Note that significantly higher concentrations of enzyme were required to degrade cellulose in rice hulls than in Whatman filter paper or hardwood. Note that $Y$-axis scales vary.

from the growth rate of different microorganisms present in the rumen fluid. While this makes interpretation more difficult, it was still possible to see that exogenous enzymes increase the digestibility of cellulose.

These results indicate that there is either endogenous cellulase activity in rumen fluid or the rumen microorganisms are synthesizing cellulase de novo. In order to differentiate whether the cellulase was due to residual activity in the rumen fluid or de novo synthesis from the microorganisms, we tested for cellulase activity before and after filter sterilization of the fluid. Filtration should remove all ruminal microorganisms, but still retain residual cel- 


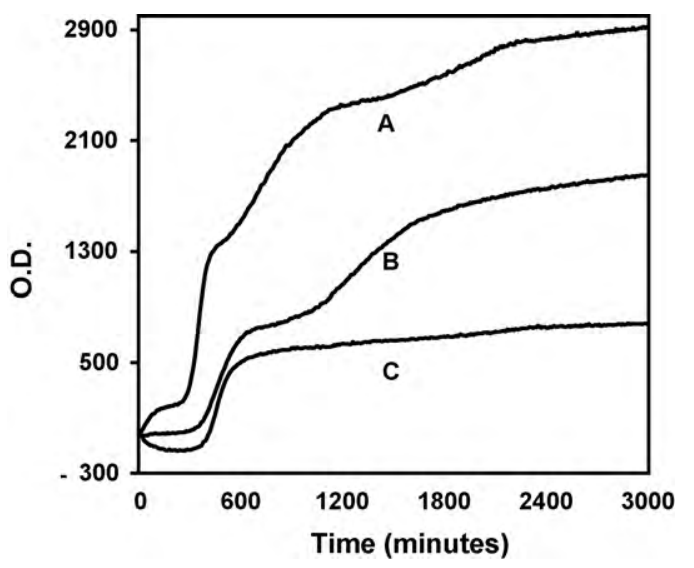

Fig. 6. Growth of rumen microorganisms on cellulose. Rumen fluid was added to cellulose substrates in assay media and growth monitored as described in Section 2. The treatments were: A: $2 \%$ glucose as a positive control; B: $1 \mathrm{~mL}$ of rumen fluid with an additional $1 \mathrm{mg}$ cellulase; $\mathrm{C}: 1 \mathrm{~mL}$ of rumen fluid with no additional cellulase Note that added cellulase supports better growth of rumen microorganisms.

lulase in the filtrate. As an additional control, rumen fluid was autoclaved to eliminate both residual cellulase activity and viable microorganisms. Samples were then tested in the BacT/Alert system and rumen microorganism growth measured between 200 and $2000 \mathrm{~min}$. Untreated rumen fluid showed growth similar to that in Fig. 6, while growth on the filtered or autoclaved rumen samples was almost completely eliminated (data not shown). Similar results were obtained using rumen fluid from two different cows. These samples were also analyzed using a biochemical assay for cellulase activity using $T$. reesei cellulase as standard; however we can only obtain a rough approximation of cellulase by this method as there are multiple forms with different specific activities. Cellulase activity of autoclaved rumen fluid was comparable to the background activity of the assay (Table 1). Cellulase activity of unfiltered fluid (non-sterile) was significantly higher than that in the filtered, microorganism-free fluid, indicating that cellulase was being synthesized de novo by rumen microorganisms during the enzyme assay. The residual activity in the rumen fluid presumably results from earlier microbial synthesis of the enzymes.

\section{Discussion}

Cellulose is notoriously difficult and slow to degrade [32,33], with reactions generally taking days, not minutes. Typically, samples are taken at discrete points during the reaction and assayed for sugar release and conversion, which are cumbersome and inconvenient. This system offers the advantage of monitoring the course of the reaction over real time, thereby providing a way of detecting changes in enzyme efficiency as a result of modifications in microcrystalline structure of cellulase or in metabolite release that affect microbial growth as the cellulose is deconstructed. With the system described above, the process can be monitored and optimal

Table 1

Effect of autoclaving and filter sterilization on rumen fluid cellulase activity.

\begin{tabular}{lc}
\hline Rumen fluid treatment & Cellulase activity in $\mathrm{mg} / \mathrm{mL}^{\mathrm{a}}$ \\
\hline Untreated control & $7.51 \pm 0.3^{\mathrm{b}}$ \\
Filter-sterilized & $5.69 \pm 0.02$ \\
Autoclaved & 0 \\
\hline
\end{tabular}

a Rumen fluid was collected and cellulase assays were done as described in Section 2 following filter sterilization or autoclaving. Untreated rumen fluid was used as control.

b Untreated rumen fluid had higher levels of cellulase than filter-sterilized fluid, indicating de novo synthesis. sampling times identified for a more detailed analysis. It should be noted that the use of the system does not accelerate the degradative process, only make it less cumbersome to monitor.

Alternative procedures can be adapted for measuring microbial growth using this basic concept. The BacT/Alert system described here does not rely on light scattering, thereby reducing the potential for interference caused by the mixture of partially digested cellulose and microorganisms. The method may prove useful for measuring the effectiveness of different enzyme mixtures, degradative microorganisms, cellulose sources, and combinations of these. In addition, the method can also be used to sample and analyze reaction products that interfere with the growth of microorganisms. Finally, the semi-automated feature can allow for multiple treatments to be done overnight.

It may be possible in the future to use this approach to estimate absolute values of cellulase, but at present, this method is limited to comparing the relative efficiencies of cellulose degradation to free sugars, thereby supporting growth. Therefore, the BacT/Alert system can be a useful tool for screening relative efficiencies of cellulose degradation, and ultimately another method such as chemical analysis of free sugars will be needed to obtain absolute quantification. For instance, comparison of cellulosic substrates indicated that rice hulls are significantly harder to degrade than hardwood or Whatman paper. This is mainly due to the presence of silica in rice hulls [34], which obstructs cellulase action. Using this system, such recalcitrance can be quickly identified amongst a variety of substrates, and the exact reason for the recalcitrance can be identified by further testing.

The purpose of this assay was to assess if enzymatic degradation of cellulose was sufficiently efficient to support the growth of fermentative organisms for downstream processes. We discovered that the system could indeed allow the establishment of optimal concentrations of enzymes that often act in unpredictable, synergistic ways, to maximize release of free sugars. Further, strains of S. cerevisiae and Zymomonas mobilis have been engineered to co-ferment both pentoses and hexoses [27,35], and future experiments can establish optimal conditions for co-cultures of cellulose-degrading and fermentative organisms to work together efficiently on varying cellulosic biomass.

The dearth of high-throughput devices to simultaneously compare multiple parameters is a bottleneck in the optimization of biomass degradation processes [36]. Since the microbial growth assay reported here is based on utilization of sugars produced, the approach is directly applicable to optimization of parameters for application to processes such as Simultaneous Saccharification and Fermentation, Simultaneous Saccharification and Co-Fermentation and Consolidated Bioprocessing [37].

\section{Disclosure}

The authors have no financial or other interest in bioMérieux the manufacturer of BacT/Alert.

\section{Acknowledgments}

This work was supported by grants from DOE (DE FG36 08G088025s), The Wal-Mart Foundation; The Walton Family Foundation; Department of the Navy, Office of Naval Research (Award \# N00014-07-1-1152). The authors would like to thank Professor Wyatt Brown, California Polytechnic State University, for the use of the HPLC. We would also like to thank Amanda Arends, Selena Tinga and Brittany Bosma who provided assistance with the experiments. Finally, we would like to thank Dr. V. Rajan for her help and editorial comments on the preparation of this manuscript. 


\section{References}

[1] Gray KA, Zhao L, Emptage M. Bioethanol. Curr Opin Chem Biol 2006;10:141-6 doi:10.1016/j.cbpa.2006.02.035 [S1367-5931(06)00037-8 [pii]].

[2] Klyosov AA. Trends in biochemistry and enzymology of cellulose degradation. Biochemistry 1990;29:10577-85.

[3] Wood TM. Fungal cellulases. Biochem Soc Trans 1992;20:46-53.

[4] Cosgrove DJ. Enzymes and other agents that enhance cell wall extensibility. Annu Rev Plant Physiol Plant Mol Biol 1999;50:391-417, doi:10.1146/annurev.arplant.50.1.391.

[5] Cantarel B, Coutinho P, Rancurel C, Bernard T, Lombard V, Henrissat B. The Carbohydrate-Active EnZymes database (CAZy): an expert resource for glycogenomics. Nucleic Acids Res 2009;37:D233.

[6] Baker J, Ehrman C, Adney W, Thomas S, Himmel M. Hydrolysis of cellulose using ternary mixtures of purified celluloses. Appl Biochem Biotechnol 1998;70:395-403.

[7] Beguin P, Aubert JP. The biological degradation of cellulose. FEMS Microbiol Rev 1994;13:25-58 [0168-6445(94)90099-X [pii]].

[8] Kabel MA, Maarel MJECvd, Klip G, Voragen AGJ, Schols HA. Standard assays do not predict the efficiency of commercial cellulase preparations towards plant materials. Biotechnol Bioeng 2006;93:56-63.

[9] Ghose T. Measurement of cellulase activities. Pure Appl Chem 1987;59:257-68

[10] Sharrock KR. Cellulase assay methods: a review. J Biochem Biophys Methods 1988;17:81-105 [0165-022X(88)90040-1 [pii]].

[11] Zhang PYH, Himmel M, Mielenz J. Outlook for cellulase improvement: screening and selection strategies. Biotechnol Adv 2006;24:452-81.

[12] Xiao Z, Storms R, Tsang A. Microplate-based filter paper assay to measure total cellulase activity. Biotechnol Bioeng 2004;88:832-7.

[13] Schmidt O, Kebernik U. A simple assay with dyed substrates to quantify cellulase and hemicellulase activity of fungi. Biotechnol Tech 1988 2:153-8.

[14] Teather R, Wood P. Use of Congo red-polysaccharide interactions in enumeration and characterization of cellulolytic bacteria from the bovine rumen. Appl Environ Microbiol 1982;43:777-80.

[15] Selig M, Weiss N, Ji Y. Enzymatic saccharification of lignocellulosic biomass. In: Laboratory analytical procedure (LAP); 2008.

[16] Canevascini G, Gattlen C. A comparative investigation of various cellulase assay procedures. Biotechnol Bioeng 1981;23:1573-90.

[17] Glasser W, Mccartney B, Samaranayake G. Cellulose derivatives with low degree of substitution. 3. The biodegradability of cellulose esters using a simple enzyme assay. Biotechnol Prog 1994;10:214-9.

[18] Huang JS, Tang J. Sensitive assay for cellulase and dextranase [cellulose]. Anal Biochem 1976;73:369-77.

[19] Kolbe J, Kubicek C. Quantification and identification of the main components of the Trichoderma cellulase complex with monoclonal antibodies using an enzyme-linked immunosorbent assay (ELISA). Appl Microbiol Biotechnol 1990;34:26-30.
[20] Béguin P. Detection of cellulase activity in polyacrylamide gels using Congo red-stained agar replicas. Anal Biochem 1983;131:333-6.

[21] Lynd L, Weimer P, van Zyl W, Pretorius I. Microbial cellulose utilization: fundamentals and biotechnology. Microbiol Mol Biol Rev 2002;66:506-77.

[22] Montenecourt B, Eveleigh D. Selective screening methods for the isolation of high yielding cellulase mutants of Trichoderma reesei. Adv Chem Ser 1979;181:289-301.

[23] Hood E, Love R, Lane J, Bray J, Clough R, Pappu K, et al. Subcellular targeting is a key condition for high-level accumulation of cellulase protein in transgenic maize seed. Plant Biotechnol J 2007;5:709-19.

[24] Gressel J. Transgenics are imperative for biofuel crops. Plant Sci 2007; 174:246-63.

[25] Sticklen M. Plant genetic engineering to improve biomass characteristics for biofuels. Curr Opin Biotechnol 2006;17:315-9, doi:10.1016/j.copbio.2006.05.003 [S0958-1669(06)00062-0 [pii]].

[26] Jeffries TW. Engineering yeasts for xylose metabolism. Curr Opin Biotechnol 2006;17:320-6, doi:10.1016/j.copbio.2006.05.008 [S0958-1669(06)00066-8 [pii]].

[27] Zhang M, Eddy C, Deanda K, Finkelstein M, Picataggio S. Metabolic engineering of a pentose metabolism pathway in ethanologenic Zymomonas mobilis. Science $1995 ; 267: 240$.

[28] Cooney CL, Wang DIC, Wang SD, Gordon J, Jiminez M. Simultaneous cellulose hydrolysis and ethanol production by a cellulolytic anaerobic bacterium; 1978.

[29] Deshpande MV. Ethanol production from cellulose by coupled saccharification/fermentation using Saccharomyces cerevisiae and cellulase complex from Sclerotium rolfsii UV-8 mutant. Appl Biochem Biotechnol 1992;36:227-34.

[30] Teunissen MJ, Op den Camp HJ. Anaerobic fungi and their cellulolytic and xylanolytic enzymes. Antonie Van Leeuwenhoek 1993;63:63-76.

[31] McAllister T, Hristov A, Beauchemin K, Rode L, Cheng K. Enzymes in ruminant diets. In: Enzymes in farm animal nutrition; 2001. pp. 273-298.

[32] Dadi A, Varanasi S, Schall C. Enhancement of cellulose saccharification kinetics using an ionic liquid pretreatment step. Biotechnol Bioeng 2006;95: 904-10.

[33] Himmel ME, Ding SY, Johnson DK, Adney WS, Nimlos MR, Brady JW, et al Biomass recalcitrance: engineering plants and enzymes for biofuels production. Science 2007;315:804-7, doi:10.1126/science.1137016 [315/5813/804 [pii]].

[34] Sainz M. Commercial cellulosic ethanol: the role of plant-expressed enzymes. In Vitro Cell Dev Biol Plant 2009;45:314-29.

[35] Hahn-Hägerdal B, Karhumaa K, Fonseca C, Spencer-Martins I, Gorwa-Grauslund M. Towards industrial pentose-fermenting yeast strains. Appl Microbiol Biotechnol 2007;74:937-53.

[36] Percival Zhang YH, Himmel ME, Mielenz JR. Outlook for cellulase improvement: screening and selection strategies. Biotechnol Adv 2006;24:452-81, doi:10.1016/j.biotechadv.2006.03.003 [S0734-9750(06)00041-3 [pii]].

[37] Taherzadeh M, Karimi K. Enzyme-based hydrolysis processes for ethanol from lignocellulosic materials: a review. BioResources 2007;2:707-38. 